\title{
Flavour in supersymmetry: horizontal symmetries or wave function renormalisation
}

\author{
Emilian Dudas, ${ }^{a, b}$ Gero von Gersdorff, ${ }^{a}$ Jeanne Parmentier ${ }^{a}$ and Stefan Pokorski ${ }^{c, 1}$ \\ ${ }^{a}$ Centre de Physique Théorique, Ecole Polytechnique and CNRS, \\ F-91128 Palaiseau, France \\ ${ }^{b}$ LPT, Bat. 210, Université de Paris-Sud, \\ F-91405 Orsay, France \\ ${ }^{c}$ Institute of Theoretical Physics, Warsaw University, \\ Hoza 69, 00-681 Warsaw, Poland \\ E-mail: Emilian.Dudas@cpht.polytechnique.fr, \\ gero.gersdorff@cpht . polytechnique.fr, \\ jeanne.parmentier@cpht.polytechnique.fr, Stefan.Pokorski@fuw.edu.pl
}

ABSTRACT: We compare theoretical and experimental predictions of two main classes of models addressing fermion mass hierarchies and flavour changing neutral currents (FCNC) effects in supersymmetry: Froggatt-Nielsen (FN) U(1) gauged flavour models and NelsonStrassler/extra dimensional models with hierarchical wave functions for the families. We show that whereas the two lead to identical predictions in the fermion mass matrices, the second class generates a stronger suppression of FCNC effects. We prove that, whereas at first sight the FN setup is more constrained due to anomaly cancelation conditions, imposing unification of gauge couplings in the second setup generates conditions which precisely match the mixed anomaly constraints in the FN setup. Finally, we provide an economical extra dimensional realisation of the hierarchical wave functions scenario in which the leptonic FCNC can be efficiently suppressed due to the strong coupling (CFT) origin of the electron mass.

KEywords: Supersymmetry Phenomenology, Phenomenology of Field Theories in Higher Dimensions

ARXIV EPRINT: 1007.5208

\footnotetext{
${ }^{1}$ Hans Fischer Senior Fellow, Institute for Advanced Studies, Technical University, Munich, Germany.
} 


\section{Contents}

1 Introduction and outline $\quad 1$

2 Horizontal symmetry versus WFR: structure and predictions 3

$\begin{array}{lll}3 & \text { Unification and wave-function hierarchies } & 9\end{array}$

4 Extra dimensional model for the WFR $\quad 11$

$\begin{array}{lll}5 & \text { Conclusions } & 14\end{array}$

\section{Introduction and outline}

The Standard Model (SM) is successful in describing the strong suppression of the FCNC and $\mathrm{CP}$ violating processes but this success strongly relies on the pattern of fermion masses and mixing angles taken from experiment. It has since long been a big theoretical challenge to find extensions of the SM that address the origin of the Yukawa couplings and simultaneously solve the hierarchy problem of the SM, in no conflict with the FCNC and $\mathrm{CP}$ violation data. The flavour structure of the new physics, needed to explain the pattern of the Yukawa matrices, also has to control the new physics at $\mathrm{TeV}$ scale that protects the Higgs potential from large radiative corrections, so that the new sources of the FCNC and $\mathrm{CP}$ violation are strongly suppressed.

It is an old and interesting proposal that the flavour dynamics and the hierarchy problem can be simultaneously addressed in supersymmetric models with spontaneously broken horizontal gauge symmetries and the Froggatt-Nielsen (FN) mechanism for Yukawa couplings [1-13]. An extensive theoretical and phenomenological work shows that such models with Abelian or non-Abelian [14-17] horizontal symmetries can correctly reproduce the pattern of Yukawa matrices. At the same time, they control the flavour structure of the soft supersymmetry breaking terms in the gravity mediation scenario and can be compatible with very strong experimental constraints from the FCNC and $\mathrm{CP}$ violation sector, without the need to raise the scale of sfermion masses beyond that needed to solve the little hierarchy problem. However, this compatibility often requires restricted range of supersymmetric parameters and/or some additional structural assumptions $[2,3,10,11,18]$ (see [19] for a recent discussion). In general, there is not much room for manoeuvre and one may expect FCNC to be close to the present bounds.

More recently, it has been proposed that the pattern of Yukawa matrices and the suppression of FCNC effects in supersymmetric theories can be understood as solely due to strong wave function renormalisation (WFR models) of the matter fields, superimposed on the initial flavour anarchical structure at the very high (Planck) scale $M_{0}$ [20]. The origin 
of such effects could be RG running down to some scale $M$ few orders of magnitude below $M_{0}$, with large anomalous dimensions of the matter fields generated by the coupling of the MSSM sector to a conformal sector [20-22] or different localisation of different matter fields in a (small) extra dimension introduced just for flavour [23-26]. The latter idea has also been extensively discussed as a solution to the flavour problem in non-supersymmetric Randall-Sundrum type models, with strongly warped extra dimensions [27-34].

As it has already been noticed in the original paper by Nelson and Strassler, the predictions of the WFR mechanism in supersymmetric models resemble those of the flavour models based on the FN mechanism, with horizontal abelian symmetries, with all SM fermions carrying charges of the same sign and with one familon field. Indeed, the predictions of the two approaches for the Yukawa matrices are identical, after proper identification of the corresponding parameters. There is a finite number of FN supersymmetric models (of the horizontal charge assignments) with abelian horizontal symmetry, one familon field and all fermion charges of the same sign that are a) theoretically consistent, b) correctly describe quark and lepton masses and mixings. Each horizontal charge assignment can be identified with concrete values of the set of free parameters in the WFR approach, with the same predictions for the Yukawa matrices. Thus, using the previous results on the FN models we can easily infer a viable set of the WFR models. We point out that this set is likely to be a complete set of such models if we require gauge coupling unification.

The magnitude of the FCNC and CP violation at the electroweak scale is determined by the coefficients of the dimension 6 operators in the effective SM lagrangian obtained after integrating out the supersymmetric degrees of freedom [35]. As we discuss below in detail, the two approaches differ significantly in their predictions for the suppression factors for some of the dimension 6 operators. It is therefore of some interest to compare other predictions, in particular for the FCNC and CP violation suppression, of the WFR models with predictions of the FN models that successfully describe fermion masses and mixing. This is the purpose of this note. It is easy to make such a comparison for each pair of the models introduced above. However, the FN models that are successful in the Yukawa sector also include models which have no correspondence to the WFR approach, like models with charges of both signs or models with several U(1)'s. Here the comparison is less straightforward but one can see some general differences.

In section 2, putting aside the potential origin of the strong WFR effects that could be responsible for the hierarchy of Yukawa couplings, we compare the structure of the operators violating flavour in the two approaches from a $4 \mathrm{~d}$ point of view, and discuss their phenomenological predictions. We draw attention to certain important structural differences in favour of WFR, such as no distinction in wave function renormalisation between fermions and antifermions, no $D$-term contribution to sfermion masses and no problem with uncontrolled coefficients of order unity. We notice that whereas significant suppression of FCNC is achieved in the squark sector, the constraints in the leptonic sector coming from $\mu \rightarrow e \gamma$ are still difficult to satisfy.

Section 3 is devoted to the discussion of the gauge coupling unification in the WFR model. A stunning coincidence is pointed out with the Green-Schwarz anomaly cancelation conditions in the horizontal symmetry models. 
In section 4 we discuss in some detail the possible origin of the strong WFR effects. For the extra dimensional interpretation, we comment on the differences and the benefits compared to RS and propose a CFT origin of the electron mass which has the virtue of decoupling the $A$-term of the electron from its Yukawa coupling. The end result is a strong suppression of the leptonic FCNC violations $(\mu \rightarrow e \gamma)$ compared to the $4 \mathrm{~d}$ discussion in section 2. Section 5 contains our conclusions.

\section{Horizontal symmetry versus WFR: structure and predictions}

We consider effective supersymmetric models with softly broken supersymmetry, described by a Kähler potential and a superpotential, below the flavour symmetry breaking scale $M$ but above the soft supersymmetry breaking scale $M_{\text {susy }}$. The flavour structure may be present in the kinetic terms, the superpotential (in general, non-renormalisable) and in the pattern of soft terms. We concentrate only on models with positive FN charges, which are relevant for the WFR case. The effective action is determined by

$$
\begin{aligned}
W= & \epsilon^{q_{i}+u_{j}+h_{u}}\left(Y_{i j}^{U}+A_{i j}^{U} X\right) Q_{i} U_{j} H_{u}+\epsilon^{q_{i}+d_{j}+h_{d}}\left(Y_{i j}^{D}+A_{i j}^{D} X\right) Q_{i} D_{j} H_{d} \\
& +\epsilon^{l_{i}+e_{j}+h_{d}}\left(Y_{i j}^{E}+A_{i j}^{E} X\right) L_{i} E_{j} H_{d} \\
K= & \epsilon^{\left|q_{i}-q_{j}\right|}\left(1+C_{i j} X^{\dagger} X\right) Q_{i}^{\dagger} Q_{j}+\cdots,
\end{aligned}
$$

where $\epsilon=\theta / M$, with $\theta$ a chiral (super)field of $\mathrm{U}(1)$ charge $-1, X=\theta^{2} F$ is the SUSY breaking spurion and all flavour matrix elements $Y_{i j}^{U}$, etc are considered to be of order one. The family charges of fermion superfields are defined as $q_{i}$ for the flavour components of the left-handed doublet $Q_{L}$, and $u_{i}$ and $d_{i}$ for the flavour components of the (left-handed) quark singlet fields $U^{c}$ and $D^{c}$, the charge conjugate of the right-handed flavour triplets $U_{R}$ and $D_{R}$, respectively, and similarly for leptons. Horizontal charges are defined in some electroweak basis. In that basis, flavour mixing is present also in the kinetic terms. However, the rotation to the canonical basis does not change the leading powers of $\epsilon$ in the rest of the lagrangian (we assume all coefficients $C_{i j}, Y_{i j}$ and $A_{i j}$ to be of $\mathcal{O}(1)$ ) and we shall always refer to the canonical basis.

In the WFR case the effective action at the scale $M$ is determined by

$$
\begin{aligned}
W= & \left(Y_{i j}^{U}+A_{i j}^{U} X\right) Q_{i} U_{j} H_{u}+\left(Y_{i j}^{D}+A_{i j}^{D} X\right) Q_{i} D_{j} H_{d} \\
& +\left(Y_{i j}^{E}+A_{i j}^{E} X\right) L_{i} E_{j} H_{d} \\
K= & \epsilon^{-2 q_{i}} Q_{i}^{\dagger} Q_{i}+C_{i j} X^{\dagger} X Q_{i}^{\dagger} Q_{j} \cdots .
\end{aligned}
$$

Here the factors $\epsilon^{-2 q_{i}}$ are the wave function renormalisation factors, originating from the physics between $M_{0}$ and $M$, and in the notation suitable for the comparison of the two approaches. Any order unity flavour mixing in the kinetic terms at the scale $M_{0}$ has already been rotated away. From a $4 \mathrm{~d}$ perspective this Lagrangian arises for example in a large- $N$ gauge theory. For smaller $N$ the dimension of the last operator could have corrections that could lead to different suppression factors. After rescaling of the wave functions $Q_{i} \rightarrow \epsilon^{q_{i}} Q_{i}$, etc (also including the possiblity of rescaling of the Higgs fields), the effective action in the 
WFR case is given by

$$
\begin{aligned}
W= & \epsilon^{q_{i}+u_{j}+h_{u}}\left(Y_{i j}^{U}+A_{i j}^{U} X\right) Q_{i} U_{j} H_{u}+\epsilon^{q_{i}+d_{j}+h_{d}}\left(Y_{i j}^{D}+A_{i j}^{D} X\right) Q_{i} D_{j} H_{d} \\
& +\epsilon^{l_{i}+e_{j}+h_{d}}\left(Y_{i j}^{E}+A_{i j}^{E} X\right) L_{i} E_{j} H_{d} \\
K= & Q_{i}^{\dagger} Q_{i}+C_{i j} \epsilon^{q_{i}+q_{j}} X^{\dagger} X Q_{i}^{\dagger} Q_{j} \cdots .
\end{aligned}
$$

The comparison of the two approaches is immediate. For the two models to give identical predictions for Yukawa couplings at the high scale the parameters of the supersymmetric WFR models are fixed in terms of the charge assignment in the FN models. However, since the wave function renormalisation does not distinguish between particles and antiparticles, the suppression of sfermion masses is much stronger in the WFR case. Similar observation in the non-SUSY case has been made in [38]. Actually the class of FN models which really compare directly to WFR models are the ones with only one $\mathrm{U}(1)_{X}$, positive charges and with only one familon field of negative charge breaking it, such that all Yukawas are generated by holomorphic couplings to the familon.

For any comparison with experimental data we have to be in the basis where the quark mass matrices are diagonal. Since the main experimental constraints come from the down quark sector it is very convenient to remain in an electroweak basis (for explicit $\mathrm{SU}(2) \times \mathrm{U}(1)$ gauge invariance) but the one in which the down quark Yukawa matrix is diagonal. Thus, the scalar field terms in the Lagrangian are subject to (appropriate) left and right rotations that diagonalise down quark Yukawa matrix. Such rotations, acting on the off-diagonal terms in the sfermion mass matrices do not change their leading suppression factors (powers of $\epsilon$ ) but generically are the source of additional contributions to the offdiagonal terms coming from the splitting in the diagonal entries. For FN models, the two obvious sources of the diagonal splitting are potentially flavour dependent order unity coefficients $C_{i i} \neq C_{j j}$ and generically present flavour dependent U(1) $D$-terms. Those additional contributions to the off-diagonal terms are of the order of the rotation angles diagonalising the down quark Yukawa matrix (roughly speaking of the order of the CKM angles) and are unwelcome. They provide an upper bound (in fact uncomfortably strong) on the suppression of the off-diagonal terms. It so happens that in the discussed here models with all fermions carrying the same sign horizontal charges the suppression factors of the original off-diagonal terms are the same as the suppression of the terms originating from the diagonal splitting so the problem of compatibility with the data is similarly difficult for both components (see [19]). However, there are U(1) models with charges of both signs and/or with several U(1) symmetries which do not have WFR counterpart but are often successful in the Yukawa sector and give strong suppression of the original flavour off-diagonal sfermion mass terms. Still, they face the mentioned above generic problem of the flavour dependent $D$-term contribution to the diagonal masses and possible diagonal splitting by uncontrolled by the U(1) symmetry coefficients of order unity. After rotations, the suppression of the off-diagonal terms in the quark mass eigenstate basis is then similar as in the models with the same sign charges and results in certain tensions in the parameter space of the soft supersymmetry breaking terms [19]. It is clear that WFR approach avoids those problems. There is no $\mathrm{U}(1)$ symmetry and no $D$-terms and the flavour dependent 
diagonal terms in the sfermion mass matrices are suppressed by powers of $\epsilon$, so there is also no problem of uncontrolled coefficients of order unity.

In addition to working in the electroweak basis with diagonal down quarks, for a meaningful comparison with experimental data we have to include all the MSSM-like renormalisation effects for the running from the scale $M$ to the electroweak scale. Finally, the standard analysis of the FCNC and CP violation data is performed in terms of the coefficients of the dimension 6 operators in the effective SM lagrangian obtained after integrating out the supersymmetric degrees of freedom [35]. The coefficients of those operators are calculable in terms of the soft supersymmetry breaking parameters and the discussed above suppression factors have a direct correspondence in the suppression factors of the higher dimension operators.

Let us compare the flavour properties of some models of fermion mass hierarchies under the two paradigms of family symmetry and WFR. From our discussion above it is clear that once we have a FN model with all fermion charges of the same sign that correctly reproduces the fermion mass hierarchy it can immediately be translated into a WFR model. ${ }^{1}$ In the following we shall compare some of the corresponding pairs of models mentioned above. As we discussed earlier, for a global picture one should also compare the set of viable WFR models with FN models that do not have any WFR correspondence but are successful in the Yukawa sector, too. However, after inclusion of the effects of the splitting on the diagonal and of the $D$-term contributions to the sfermion masses such models give predictions for FCNC very close to the same sign charge models, so we don't discuss them any more. From the point of view of proton decay operators, both approaches can generate some suppression: $\mathrm{U}(1)_{X} \mathrm{FN}$ can also completely kill proton decay if for example the lepton charges are $l_{i}=n_{i}+1 / 3, e_{i}=m_{i}-1 / 3$, with $n_{i}, m_{i}$ integers (all other MSSM charges being integers), since then there is an effective $Z_{3}$ discrete leptonic symmetry protecting the proton to decay. More generally, both FN and WFR generate some suppression for the first generations due to their large charges.

The flavour suppression is parameterised by the variable $\epsilon$ introduced earlier. We have in mind $\epsilon$ to be of the order the Cabbibo angle, $\epsilon \sim 0.22$, but certainly other values can be considered provided one appropriately rescales the charges. Consistent charge assignments have for instance been classified in refs. [8, 9, 12]. Here we will consider 3 models: $^{2}$

$$
\begin{array}{llrlrl}
q=u=e=(3,2,0), & d=\ell=(2,0,0)+d_{3}, & & \text { (Model A) } \\
q=u=e=(4,2,0), & d=\ell=(1,0,0)+d_{3}, & & \text { (Model B) } \\
q=(3,2,0), \quad u=(5,2,0), & d=(1,0,0)+d_{3}, \quad \ell=q+\ell_{3}, \quad e=d-\ell_{3} . & \text { (Model C) }
\end{array}
$$

In all three cases the horizontal charges of the Higgs fields are zero. Notice that the choice $q_{3}=u_{3}=0$ is a requirement for obtaining a heavy top, while the freedom in $d_{3}$ is related

\footnotetext{
${ }^{1} \mathrm{FN}$ are seemingly more constrained, as some form of anomaly cancelation has to be imposed. We will see in section 3 that preservation of the successful MSSM gauge coupling unification in WFR models places very similar constraints on the assignment of "charges" (suppression factors) in the latter case.

${ }^{2} \mathrm{~A}$ and $\mathrm{B}$ are taken from ref. [12], where they are called models 1 and 5 respectively, model $\mathrm{C}$ was studied in ref. [8, 9].
} 


\begin{tabular}{|c|c|c|c|}
\hline$a$ & $\tilde{m}_{a, L L}^{2} / m_{0}^{2}$ & $\tilde{m}_{a, R R}^{2} / m_{0}^{2}$ & $A_{a} / m_{0} \sim Y_{a}$ \\
\hline$u$ & $\begin{array}{l}r_{q} \mathbf{1}+\left(\begin{array}{ccc}\epsilon^{6} & \epsilon^{5} & \epsilon^{3} \\
\epsilon^{5} & \epsilon^{4} & \epsilon^{2} \\
\epsilon^{3} & \epsilon^{2} & \epsilon^{0}\end{array}\right) \\
r_{q} \mathbf{1}+\left(\begin{array}{ccc}\epsilon^{0} & \epsilon^{1} & \epsilon^{3} \\
\epsilon^{1} & \epsilon^{0} & \epsilon^{2} \\
\epsilon^{3} & \epsilon^{2} & \epsilon^{0}\end{array}\right)\end{array}$ & $\begin{array}{r}r_{u} \mathbf{1}+\left(\begin{array}{ccc}\epsilon^{6} & \epsilon^{5} & \epsilon^{3} \\
\epsilon^{5} & \epsilon^{4} & \epsilon^{2} \\
\epsilon^{3} & \epsilon^{2} & \epsilon^{0}\end{array}\right) \\
r_{u} \mathbf{1}+\left(\begin{array}{ccc}\epsilon^{0} & \epsilon^{1} & \epsilon^{3} \\
\epsilon^{1} & \epsilon^{0} & \epsilon^{2} \\
\epsilon^{3} & \epsilon^{2} & \epsilon^{0}\end{array}\right)\end{array}$ & $\left(\begin{array}{ccc}\epsilon^{6} & \epsilon^{5} & \epsilon^{3} \\
\epsilon^{5} & \epsilon^{4} & \epsilon^{2} \\
\epsilon^{3} & \epsilon^{2} & \epsilon^{0}\end{array}\right)$ \\
\hline$d$ & $\begin{array}{l}r_{q} \mathbf{1}+\left(\begin{array}{ccc}\epsilon^{6} & \epsilon^{5} & \epsilon^{3} \\
\epsilon^{5} & \epsilon^{4} & \epsilon^{2} \\
\epsilon^{3} & \epsilon^{2} & \epsilon^{0}\end{array}\right) \\
r_{q} \mathbf{1}+\left(\begin{array}{ccc}\epsilon^{0} & \epsilon^{1} & \epsilon^{3} \\
\epsilon^{1} & \epsilon^{0} & \epsilon^{2} \\
\epsilon^{3} & \epsilon^{2} & \epsilon^{0}\end{array}\right)\end{array}$ & 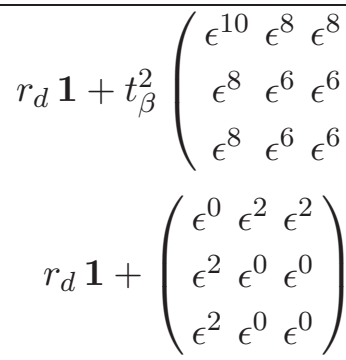 & $t_{\beta}\left(\begin{array}{ccc}\epsilon^{8} & \epsilon^{6} & \epsilon^{6} \\
\epsilon^{7} & \epsilon^{5} & \epsilon^{5} \\
\epsilon^{5} & \epsilon^{3} & \epsilon^{3}\end{array}\right)$ \\
\hline$e$ & $\begin{array}{r}r_{\ell} \mathbf{1}+t_{\beta}^{2}\left(\begin{array}{ccc}\epsilon^{10} & \epsilon^{8} & \epsilon^{8} \\
\epsilon^{8} & \epsilon^{6} & \epsilon^{6} \\
\epsilon^{8} & \epsilon^{6} & \epsilon^{6} \\
r_{\ell} \mathbf{1}+\left(\begin{array}{ccc}\epsilon^{0} & \epsilon^{2} & \epsilon^{2} \\
\epsilon^{2} & \epsilon^{0} & \epsilon^{0} \\
\epsilon^{2} & \epsilon^{0} & \epsilon^{0}\end{array}\right)\end{array}\right.\end{array}$ & $\begin{array}{l}r_{e} \mathbf{1}+\left(\begin{array}{ccc}\epsilon^{6} & \epsilon^{5} & \epsilon^{3} \\
\epsilon^{5} & \epsilon^{4} & \epsilon^{2} \\
\epsilon^{3} & \epsilon^{2} & \epsilon^{0}\end{array}\right) \\
r_{e} \mathbf{1}+\left(\begin{array}{ccc}\epsilon^{0} & \epsilon^{1} & \epsilon^{3} \\
\epsilon^{1} & \epsilon^{0} & \epsilon^{2} \\
\epsilon^{3} & \epsilon^{2} & \epsilon^{0}\end{array}\right)\end{array}$ & $t_{\beta}\left(\begin{array}{ccc}\epsilon^{8} & \epsilon^{7} & \epsilon^{5} \\
\epsilon^{6} & \epsilon^{5} & \epsilon^{3} \\
\epsilon^{6} & \epsilon^{5} & \epsilon^{3}\end{array}\right)$ \\
\hline
\end{tabular}

Table 1. Yukawas and soft scalar mass squared matrices for model A [12]: $q=u=e=(3,2,0)$, $\ell=d$ with $d-d_{3}=(2,0,0)$ and we have used the relation $\tan \beta=\epsilon^{d_{3}-3}$. The upper row corresponds to a WFR model, while the lower one to a FN one.

to $\tan \beta$ via the bottom Yukawa:

$$
\epsilon^{-d_{3}} \tan \beta \sim \frac{m_{t}\left(M_{c}\right)}{m_{b}\left(M_{c}\right)} \sim \epsilon^{-3} .
$$

The resulting Yukawa couplings for model A are displayed in the last column of table 1 . They readily reproduce the observed masses and mixings of the SM fermions for suitable choices of $O(1)$ coefficients.

After inclusion of the renormalisation effects [36], the soft mass terms at the elec- 
troweak scale are to a good approximation given by

$$
\begin{aligned}
\tilde{m}_{u, L L, i j}^{2} & \sim r_{q} m_{1 / 2}^{2} \delta_{i j}+\hat{m}_{q}^{2} \epsilon^{\left|q_{i} \pm q_{j}\right|} \\
\tilde{m}_{u, R R, i j}^{2} & \sim r_{u} m_{1 / 2}^{2} \delta_{i j}+\hat{m}_{u}^{2} \epsilon^{\left|u_{i} \pm u_{j}\right|} \\
\tilde{m}_{u, L R, i j}^{2} & \sim A_{u} v \sin \beta \epsilon^{q_{i}+u_{j}} \\
\tilde{m}_{d, L L, i j}^{2} & \sim r_{q} m_{1 / 2}^{2} \delta_{i j}+\hat{m}_{q}^{2} \epsilon^{\left|q_{i} \pm q_{j}\right|} \\
\tilde{m}_{d, R R, i j}^{2} & \sim r_{d} m_{1 / 2}^{2} \delta_{i j}+\hat{m}_{d}^{2} \epsilon^{\left|d_{i} \pm d_{j}\right|} \\
\tilde{m}_{d, L R, i j}^{2} & \sim A_{d} v \cos \beta \epsilon^{q_{i}+d_{j}} \\
\tilde{m}_{e, L L, i j}^{2} & \sim r_{\ell} m_{1 / 2}^{2} \delta_{i j}+\hat{m}_{\ell}^{2} \epsilon^{\left|\ell_{i} \pm \ell_{j}\right|} \\
\tilde{m}_{e, R R, i j}^{2} & \sim r_{e} m_{1 / 2}^{2} \delta_{i j}+\hat{m}_{e}^{2} \epsilon^{\left|e_{i} \pm e_{j}\right|} \\
\tilde{m}_{e, L R, i j}^{2} & \sim A_{e} v \cos \beta \epsilon^{\ell_{i}+e_{j}}
\end{aligned}
$$

where we have defined the high scale soft masses $m_{1 / 2}, \hat{m}_{a}$ and $A_{a}$. Baring some additional suppression mechanism, a completely natural theory would require all these terms to be of the same order, and we will henceforth set them all equal to a common mass $m_{0}$. The terms that are suppressed by powers of $\epsilon$ are multiplied by flavour dependent $\mathcal{O}(1)$ coefficients that are omitted here for clarity. The charges are all positive or zero, and the positive sign applies to the WFR case whereas the negative one to the FN case. The constants $r_{a}$ parameterize the leading gauge renormalization and are given approximately by $r_{q}=6.5$, $r_{u}=6.2, r_{d}=6.1, r_{\ell}=0.5$ and $r_{e}=0.15$. Yukawa corrections are expected to be important for the third generation but given the unknown $\mathcal{O}(1)$ coefficients of the tree level soft mass matrices they are irrelevant for our discussion. The resulting soft mass matrices are also displayed in table 1. Several points deserve to be emphasized.

- For WFR all 1st and 2nd generation mass eigenvalues (and, in fact, some 3rd generation ones as well) are predicted from the running of gauge/gaugino loops, while in the FN case the explicit tree level soft masses give non-negligible contribution, particularly to slepton masses.

- Yukawas and chirality changing soft masses ( $A$-terms) receive the same suppression, and are in fact the same for both FN and WFR.

- In the LL and RR sectors the off-diagonal masses are more suppressed for WFR than for FN, as explained above.

To compare with experiment, bounds are usually given for the mass insertion parameters $\delta_{i j}^{a}$ at a reference sfermion mass. They are defined as

$$
\delta_{M N, i j}^{a}=\frac{\tilde{m}_{a, M N, i j}^{2}}{\tilde{m}_{a, M, i} \tilde{m}_{a, N, j}}, \quad\left\langle\delta_{i j}^{a}\right\rangle=\sqrt{\delta_{L L, i j}^{a} \delta_{R R, i j}^{a}}
$$

for $a=u, d, e, M, N=L, R, i \neq j$. The expressions are normalized to the diagonal entries $\tilde{m}_{a, M, i}$. To the $A$ terms one associates analogous parameters (for any $i, j$ ):

$$
\delta_{L R, i j}^{a}=\left(\delta_{R L, j i}^{a}\right)^{*}=\frac{\tilde{m}_{a, L R, i j}^{2}}{\tilde{m}_{a, L, i} \tilde{m}_{a, R, j}} .
$$




\begin{tabular}{|cc|cc|cc|cc|}
\hline$a$ & $i j$ & \multicolumn{2}{|c|}{$\delta_{L L, i j}^{a}$} & \multicolumn{2}{|c|}{$\delta_{R R, i j}^{a}$} & \multicolumn{2}{c|}{$\left\langle\delta_{i j}^{a}\right\rangle$} \\
& & Exp. & Th & Exp. & Th. & Exp. & Th. \\
\hline$d$ & 12 & 0.03 & $8.6 \times 10^{-5}$ & 0.03 & $9.1 \times 10^{-7} t_{\beta}^{2}$ & 0.002 & $8.9 \times 10^{-6} t_{\beta}$ \\
$d$ & 13 & 0.2 & $1.8 \times 10^{-3}$ & 0.2 & $9.1 \times 10^{-7} t_{\beta}^{2}$ & 0.07 & $4.0 \times 10^{-6} t_{\beta}$ \\
$d$ & 23 & 0.6 & $8.1 \times 10^{-3}$ & 1.8 & $1.8 \times 10^{-5} t_{\beta}^{2}$ & 0.2 & $3.8 \times 10^{-4} t_{\beta}$ \\
\hline$u$ & 12 & 0.1 & $8.6 \times 10^{-5}$ & 0.1 & $8.6 \times 10^{-5}$ & 0.008 & $8.6 \times 10^{-5}$ \\
\hline
\end{tabular}

Table 2. Bounds on hadronic chirality-preserving mass insertions and results from WFR with model A. Bounds (taken from table IV of ref. [39]) are valid for a squark mass of $1 \mathrm{TeV}$ and scale linearly with the latter.

\begin{tabular}{|cc|cc|cc|}
\hline$a$ & $i j$ & \multicolumn{2}{|c|}{$\delta_{L R, i j}^{a}$} & \multicolumn{2}{c|}{$\delta_{R L, i j}^{a}$} \\
& & Exp. & Th. & Exp. & Th. \\
\hline$d$ & 12 & $2 \times 10^{-4}$ & $8.1 \times 10^{-6}$ & $2 \times 10^{-3}$ & $1.8 \times 10^{-6}$ \\
$d$ & 13 & 0.08 & $8.1 \times 10^{-6}$ & 0.08 & $3.7 \times 10^{-5}$ \\
$d$ & 23 & 0.01 & $3.7 \times 10^{-5}$ & 0.01 & $7.6 \times 10^{-4}$ \\
$d$ & 11 & $4.7 \times 10^{-6}$ & $3.9 \times 10^{-7}$ & $4.7 \times 10^{-6}$ & $3.9 \times 10^{-7}$ \\
\hline$u$ & 12 & 0.02 & $3.7 \times 10^{-5}$ & 0.02 & $3.7 \times 10^{-5}$ \\
$u$ & 11 & $9.3 \times 10^{-6}$ & $8.1 \times 10^{-6}$ & $9.3 \times 10^{-6}$ & $8.1 \times 10^{-6}$ \\
\hline
\end{tabular}

Table 3. Bounds on hadronic chirality-flipping mass insertions and results from WFR with model A. Bounds taken from table $\mathrm{V}$ of ref. [39] are valid for a squark mass of $1 \mathrm{TeV}$. While the bounds on the $i \neq j(i=j)$ elements grow linearly (quadratically) with the latter, our predictions go down linearly.

Starting with the limits from the hadron sector, we give the bounds and the results for Model A in table 2 and 3. All bounds in table 2 are comfortably satisfied (even for large $\tan \beta$ ) and in fact would allow for a much smaller squark mass. Notice that in the FN model with analogous charge assignments it is very difficult to satify the bound on $\left\langle\delta_{12}\right\rangle$ [19]. Since the squark mass mixing between the first two generations is suppressed at most by two powers of $\epsilon$, to satisfy the bound one needs very strong flavour blind renormalisation effects, i.e. a large ratio of of the initial values of the gluino mass to the squark mass at the very high scale.

The chirality flipping mass insertions of Tab 3 are more constraining. In particular, the 11 entries are strongly constrained from the EDM measurements of the neutron. Nevertheless, the corresponding predictions of our model for $1 \mathrm{TeV}$ squark mass marginally satisfy the experimental bounds.

Turning to leptons, we quote in table 4 the bounds ${ }^{3}$ resulting from LFV decays of the charged leptons, $\mu \rightarrow e \gamma, \tau \rightarrow e \gamma$ and $\tau \rightarrow \mu \gamma$ and the theoretical predictions ob-

\footnotetext{
${ }^{3}$ Note that the decay rate depends on the sum $\left(\delta_{L R, i j}\right)^{2}+\left(\delta_{R L, i j}\right)^{2}[35]$. The $L L$ and $R R$ entries are much less constrained and we will not consider them here.
} 


\begin{tabular}{|c|ccc|}
\hline$i j$ & \multicolumn{3}{|c|}{$\delta_{M N, i j}^{e}$} \\
& Exp. & Th. (LR) & Th. (RL) \\
\hline 12 & $4.8 \times 10^{-6}$ & $2.0 \times 10^{-5}$ & $9.4 \times 10^{-5}$ \\
13 & $1.8 \times 10^{-2}$ & $4.3 \times 10^{-4}$ & $9.4 \times 10^{-5}$ \\
23 & $1.2 \times 10^{-2}$ & $8.9 \times 10^{-3}$ & $4.3 \times 10^{-4}$ \\
\hline
\end{tabular}

Table 4. Experimental bounds on leptonic mass insertions and results from WFR with model A. Bounds (taken from table 7 of ref. [35], using updated bounds on the branching ratios [40]) are valid for a slepton mass of $400 \mathrm{GeV}$.

tained under the assumption of a universal supersymmetry breaking scale $m_{0}$ at high energy. Then, at the electroweak scale $A_{e} \sim m_{0}$ and the typical scale for sleptons is $\tilde{m}_{s l}=\left(r_{\ell} r_{e}\right)^{\frac{1}{4}} m_{0}$. One observes that even for the slepton mass as high as $400 \mathrm{GeV}$ (corresponding the $m_{0}=750 \mathrm{GeV}$ ) the contribution to $\mu \rightarrow e \gamma$ is not sufficiently suppressed. It is interesting to know how far one can adjust the charges $e_{i}$ and $\ell_{i}$ to ameliorate this problem. To this end, consider the product

$$
\delta_{L R, 12}^{e} \delta_{R L, 12}^{e} \sim \frac{A_{e}^{2} v^{2}}{\tilde{m}_{s l}^{4}} \epsilon^{\ell_{1}+e_{2}+\ell_{2}+e_{1}} \cos ^{2} \beta \sim \frac{A_{e}^{2} m_{e} m_{\mu}}{\tilde{m}_{s l}^{4}} .
$$

It is therefore clear that this product is independent of the concrete charge assignment and can only be lowered by increasing $\tilde{m}_{s l}$ or decreasing $A_{e}$. This means that at least one of the individual contributions is bigger than

$$
\frac{A_{e} \sqrt{m_{e} m_{\mu}}}{\tilde{m}_{s l}^{2}} \sim 3.5 \times 10^{-5}
$$

where the numbers are for $\tilde{m}_{s l}=400 \mathrm{GeV}$. This is a robust prediction (up to $\mathcal{O}(1)$ coefficients), and indeed table 4 shows that it holds in particular for model $A$. A stronger suppression can be obtained only if $A$ terms are smaller than $m_{0}$ and/or $m_{0}$ has larger value, i.e. $\tilde{m}_{s l}>400 \mathrm{GeV}$. For instance one can get an acceptable decay rate for $A_{e} \sim 100 \mathrm{GeV}$ and $\tilde{m}_{s l} \sim 400 \mathrm{GeV}$. Lowering the slepton mass further requires more and more fine tuning of $A_{0}$, while $\tilde{m}_{s l} \sim 400 \mathrm{GeV}$ implies squark masses of the order of $1.9 \mathrm{TeV}$ which is uncomfortably large for the little hierarchy problem. In conclusion, the leptonic bounds are more constraining than the hadronic ones ( see also [20, 25, 26]). Finally, let us stress that FN models do possess a similar problem (with identical bounds on the $L R / R L$ sector). In addition, they predict insufficient suppression in the $L L$ and $R R$ sectors.

In section 4 we will point out a novel mechanism to suppress the $\mu \rightarrow e \gamma$ decay rate, opening the possibility to lower the superpartner masses without fine-tuning the leptonic $A$ terms in the WFR models.

\section{Unification and wave-function hierarchies}

The physical gauge coupling in a supersymmetric field theory is given by [42, 43]

$$
\frac{4 \pi^{2}}{g_{a}^{2}(\mu)}=R e f_{a}+\frac{b_{a}}{4} \log \frac{\Lambda^{2}}{\mu^{2}}+\frac{T\left(G_{a}\right)}{2} \log g_{a}^{-2}\left(\mu^{2}\right)-\sum_{r} \frac{T_{a}(r)}{2} \log \operatorname{det} Z_{(r)}\left(\mu^{2}\right),
$$


where

$$
b_{a}=\sum_{r} n_{r} T_{a}(r)-3 T\left(G_{a}\right) \quad, \quad T_{a}(r)=\operatorname{Tr}_{r} T_{(a)}^{2}
$$

are the beta function and the Dynkin index of the representation $r$ under the gauge group factor $G_{a}, f_{a}$ are the holomorphic gauge couplings, $Z_{(r), i j}$ are wave functions of matter fields of flavour indices $i, j$ and the determinant $\operatorname{det} Z_{(r)}\left(\mu^{2}\right)$ is taken in the flavour space. In our case $Z_{(r)} \simeq \operatorname{diag}\left(\epsilon^{-2 q_{1}^{(r)}}, \epsilon^{-2 q_{2}^{(r)}}, \epsilon^{-2 q_{3}^{(r)}}\right)$ and therefore

$$
\log \operatorname{det} Z_{(r)}=-2 \sum_{i} q_{i}^{(r)} \log \epsilon
$$

where $q_{i}^{(r)}$ are the " $\mathrm{U}(1)$ charges" of the matter representations $r=Q, U, D, L, E, H_{u}, H_{d}$. Let us define in what follows the quantities

$$
A_{a}=-\frac{1}{\log \epsilon} \sum_{r} \frac{T_{a}(r)}{2} \log \operatorname{det} Z_{(r)},
$$

which are proportional to the additional contribution to the running coming from a strongly coupled sector, producing the hierarchical wave functions. Notice that usual MSSM unification is preserved if

$$
A_{3}=A_{2}=\frac{3}{5} A_{1}
$$

With the field content of MSSM, we find

$$
\begin{array}{ll}
\mathrm{SU}(3): & A_{3}=\sum_{i}\left(2 q_{i}+u_{i}+d_{i}\right), \\
\mathrm{SU}(2): & A_{2}=\sum_{i}\left(3 q_{i}+l_{i}\right)+h_{u}+h_{d}, \\
\mathrm{U}(1)_{Y}: & A_{1}=\sum_{i}\left(\frac{1}{3} q_{i}+\frac{8}{3} u_{i}+\frac{2}{3} d_{i}+l_{i}+2 e_{i}\right)+h_{u}+h_{d} .
\end{array}
$$

Notice also that the quantities $A_{i}$ can be related simply to the determinants of the mass matrices of the quarks and leptons via

$$
\begin{aligned}
\operatorname{det}\left(Y_{U} Y_{D}^{-2} Y_{L}^{3}\right) & =\epsilon^{\frac{3}{2}\left(A_{1}+A_{2}-2 A_{3}\right)}, \\
\operatorname{det}\left(Y_{U} Y_{D}\right) & =\epsilon^{A_{3}+3\left(h_{u}+h_{d}\right)} .
\end{aligned}
$$

The reader familiar with the gauged Froggatt-Nielsen U(1) generating Yukawa hierarchies probably recognized already (3.5)-(3.7), see [4-9]. It is worth pointing out the interesting analogy with our present case:

- In the gauged FN case the quantities (3.6) are precisely the coefficients of the $\mathrm{U}(1)_{X} G_{a}^{2}$ mixed anomalies, between the gauged $\mathrm{U}(1)_{X}$ and the SM gauge group factors $G_{a}=\mathrm{SU}(3), \mathrm{SU}(2), \mathrm{U}(1)_{Y}$.

- In the FN case (3.5) represent the universal (for the heterotic strings) Green-Schwarz anomaly cancelation conditions. 
In our case, (3.5) represent the unification conditions for the gauge couplings at the energy scale where the strong sector decouples from the running. Interestingly enough, even if there is no gauge $\mathrm{U}(1)$ symmetry in our case, unification of gauge couplings requires the "charges" determining the wave function renormalisation to satisfy exactly the same constraints as anomaly cancellation for the U(1) charges in the gauged FN case! Notice that models $\mathrm{A}$ and $\mathrm{B}$ defined in section 2 trivially satisfy eq. (3.5) as the charges are $\mathrm{SU}(5)$ invariant, whereas model $\mathrm{C}$ is a nontrivial solution. This stunning coincidence of the anomaly cancelation conditions and the conditions for the gauge coupling unification can be qualitatively related in the following way. The dimensions of the operators are linked to the charges under the $\mathrm{U}(1)_{R}$ current, partner of the dilatation current. On the other hand, the anomalies $\mathrm{U}(1)_{R} G_{a}^{2}$ are indeed related to the beta functions and therefore to the running of the gauge couplings of the SM gauge factors $G_{a}$ (for a related discussion see ref. [44]).

By using the results of [5-9] on the structure of quark and lepton masses, one useful relation can be written

$$
A_{1}+A_{2}-\frac{8}{3} A_{3} \simeq 2\left(h_{u}+h_{d}\right) .
$$

The unification conditions (3.5) lead therefore to $h_{u}+h_{d}=0$. Since in the WFR models all "charges" are positive or zero, this means that $h_{u}=h_{d}=0$. Therefore in the extra dimensional interpretation of the WFR models (to be discussed in the next section) both Higgs doublets are localized on the UV brane. Let us mention that another plausible possibility for unification is that the holomorphic gauge couplings and the wave function contributions separately violate the SU(5) gauge coupling relation but their combined contributions preserve it. For detailed studies of gauge coupling unifications in theories with strongly coupled sectors see ref. [45-48].

Let us notice that, in the FN case, the mixed anomaly conditions (3.6) imposed to the model $\mathrm{C}$ of section (2) gives the result $h_{u}+h_{d}=0, d_{3}-l_{3}=2 / 3$. A simple solution is $h_{u}=h_{d}=0, d_{3}=1, l_{3}=1 / 3$. In this case, the $\mathrm{U}(1)_{X}$ symmetry breaks to a discrete $Z_{3}^{L}$ acting on the leptons, which protects proton decay.

\section{Extra dimensional model for the WFR}

There are various possible origins for the WFR: 4d strongly coupled or higher-dimensional with flavour-dependent wave-function localization. We use here a variant of the RS setup [49], with an UV brane with energy scale $\Lambda_{\mathrm{UV}}$ and an IR brane with energy scale $\Lambda_{\mathrm{IR}} \sim M_{\mathrm{GUT}}$. The fifth dimension is therefore very small and the hierarchy is given by

$$
\epsilon=\frac{\Lambda_{\mathrm{IR}}}{\Lambda_{\mathrm{UV}}}=e^{-k \pi R}
$$

All MSSM fields live in the bulk [50]. Following [23, 24], start with Kähler terms $(0<y<\pi R)$

$$
\begin{aligned}
\hat{K}= & e^{\left(1-2 c_{h_{u}}\right) k y} H_{u}^{\dagger} H_{u}+e^{\left(1-2 c_{h_{d}}\right) k y} H_{d}^{\dagger} H_{d} \\
& +e^{\left(1-2 c_{q, i}\right) k y} Q_{i}^{\dagger} Q_{i}+e^{\left(1-2 c_{u_{i}}\right) k y} U_{i}^{\dagger} U_{i}+e^{\left(1-2 c_{d_{i}}\right) k y} D_{i}^{\dagger} D_{i} \\
& +\delta(y) k^{-3} X^{\dagger} X\left(C_{q, i j} Q_{i}^{\dagger} Q_{i}+C_{u, i j} U_{i}^{\dagger} U_{i}+C_{d, i j} D_{i}^{\dagger} D_{i}+C_{h_{u}} H_{u}^{\dagger} H_{u}+C_{h_{d}} H_{d}^{\dagger} H_{d}\right) .
\end{aligned}
$$


where $i, j=1,2,3$ running over families and the coefficients are flavour anarchic $\mathcal{O}(1)$ numbers. We have kept only fields with zero modes, the conjugate fields $\phi^{c}$ have Dirichlet boundary conditions (--) and hence have no zero modes. The leptons have an analogous Lagrangian. Brane localized kinetic terms can also be introduced, even with arbitrary flavour dependence, without changing the outcome. We will introduce a superpotential

$$
\begin{aligned}
\hat{W}= & \delta(y) k^{-\frac{3}{2}}\left(\hat{Y}_{i j}^{u} H_{u} Q_{i} U_{j}+\hat{Y}_{i j}^{d} H_{d} Q_{i} D_{j}+k^{-1} X \hat{A}_{i j}^{u} H_{u} Q_{i} U_{j}+k^{-1} X \hat{A}_{i j}^{d} H_{d} Q_{i} D_{j}\right) \\
& +\delta(y-\pi R)(k \epsilon)^{-\frac{3}{2}}\left(\hat{Y}_{i j}^{\prime u} \epsilon^{c_{h_{u}}+c_{q_{i}}+c_{u_{j}}} H_{u} Q_{i} U_{j}+\hat{Y}_{i j}^{\prime d} \epsilon^{c_{h_{d}}+c_{q_{i}}+c_{d_{j}}} H_{d} Q_{i} D_{j}\right)
\end{aligned}
$$

Notice that we have confined the SUSY breaking spurion $X$ to the UV brane at $y=0$. We have introduced arbitrary dimensionless Yukawa couplings on both branes. After integrating over the extra dimension, the kinetic terms pick up wave function renormalisations

$$
Z_{q}=\frac{1}{\left(1-2 c_{q}\right) k}\left(\epsilon^{2 c_{q}-1}-1\right)
$$

and therefore

$$
Z_{q} \sim \frac{\epsilon^{2 c_{q}-1}}{\left(1-2 c_{q}\right) k} \text { for } c<1 / 2 \text { and } Z_{q} \sim \frac{1}{\left(2 c_{q}-1\right) k} \text { for } c>1 / 2 .
$$

Notice that

- For $c_{q}<\frac{1}{2}$ the field is localized near the IR brane. We assign it "charges" $q=\frac{1}{2}-c_{q}>$ 0 and $q^{\prime}=0$.

- For $c_{q}>\frac{1}{2}$ the field is localized near the UV brane. The charges are $q=0$ and $q^{\prime}=c_{q}-\frac{1}{2}>0$.

- Exact UV (IR) brane localization is obtained by formally sending $q^{\prime}(q)$ to infinity.

After switching to canonical normalization, this leads to Yukawa couplings

$$
\begin{aligned}
& Y_{i j}^{u}=\hat{Y}_{i j}^{u} \epsilon^{q_{i}+u_{j}+h_{u}}+\hat{Y}_{i j}^{\prime u} \epsilon^{q_{i}^{\prime}+u_{j}^{\prime}+h_{u}^{\prime}}, \\
& Y_{i j}^{d}=\hat{Y}_{i j}^{d} \epsilon^{q_{i}+d_{j}+h_{d}}+\hat{Y}_{i j}^{\prime d} \epsilon^{q_{i}^{\prime}+d_{j}^{\prime}+h_{d}^{\prime}} .
\end{aligned}
$$

Each field either suppresses $\hat{Y}$ or $\hat{Y}^{\prime}$, depending on whether it is UV or IR localized.

Since we will take the $X$ field localized on the UV brane, the physical soft masses and $A$ terms at the high scale are given by the expressions in section 2 with

$$
m_{0} \sim \frac{\left|F_{X}\right|}{k} .
$$

We consider the following localisation of the MSSM fields: ${ }^{4}$

- the first two generations of quarks and leptons are localized near the IR brane. In a holographic $4 \mathrm{~d}$ interpretation, the first two generations are composite states.

\footnotetext{
${ }^{4}$ Similar localization of the MSSM flavours from a different perspective was also considered recently in $[52,53]$.
} 
- the top quark is localized on or near the UV brane, whereas bottom and tau are localized near the UV brane or near the IR brane, depending on $\tan \beta$. In the holographic language, the top quark is therefore elementary.

- the two Higgs doublets $H_{u}, H_{d}$ are localized near the UV brane and therefore have $h_{u}, h_{d}=0$. They are then elementary from the $4 \mathrm{~d}$ holographic point of view. In the scenario below, we will consider a finite $h_{d}^{\prime}$ describing a non-negligible "tail" near the IR.

- the spurion $X$ is located on the UV brane

One important point to mention here is that the extra dimensional realisation of the WFR approach allows for certain generalisations. They are equivalent (and the analogy with FN is true) only if all Yukawas are localized on the UV brane that is if we neglect the corrections coming from the "tail" of the Higgs fields near the IR brane.

By comparing with the standard RS non-SUSY setup with fermion mass hierarchies generated by wave functions overlap, we notice that in the standard RS case, since $\epsilon_{\mathrm{RS}}=$ $\frac{\Lambda_{\mathrm{UV}}}{\Lambda_{\mathrm{IR}}} \sim 10^{-16}$, the bulk masses $c_{i}$ have to be tuned close to $1 / 2$ in order not to generate too big hierarchies in the fermion masses. In our case, we choose to work with a very small extra dimension $10^{-3} \leq \epsilon \leq 10^{-1}$ and therefore there is no need for such a tuning. Of course, such a small warping does not provide a solution to the hierarchy problem anymore, but since we have low-energy supersymmetry, the strong warping is clearly not needed.

Provided $h_{u}^{\prime}$ and $h_{d}^{\prime}$ are large enough (sharp UV localization), the Yukawa couplings originating from the IR brane (i.e. the terms proportional to $\hat{Y}^{\prime}$ in eqs. (4.6) and (4.7)) are always subleading compared to the ones from the UV brane and hence irrelevant. For moderately large values they can become comparable,$^{5}$ at least for the light generations, and can in fact be exploited to circumvent the $\mu \rightarrow e \gamma$ problem pointed out at the end of section 2. For instance, for all 3 generations of leptons IR-localized (small to moderate $\tan \beta$ ), one has

$$
Y_{i j}^{e}=\hat{Y}_{i j}^{e} \epsilon^{\ell_{i}+e_{j}}+\hat{Y}_{i j}^{\prime e} \epsilon^{h_{d}^{\prime}}, \quad A_{i j}^{e}=m_{0} \hat{A}_{i j}^{e} \epsilon^{\ell_{i}+e_{j}}
$$

Ideally, we would like to suppress the dangerous $A$ terms without suppressing the corresponding Yukawas. This is easy to do: Let us imagine that we increase $\ell_{1}$ and/or $e_{1}$ such that $A_{12}^{e}$ and $A_{21}^{e}$ are sufficiently small in order to satisfy the bounds for a given slepton mass. ${ }^{6}$ Of course, this will result in a too small electron mass unless we impose that $h_{d}^{\prime}$ is responsible for generating $Y_{11}^{e}$ from the IR brane. We thus choose charges such that

$$
\begin{aligned}
\ell_{1}+e_{1} & >h_{d}^{\prime} \\
h_{d}^{\prime} & \sim 5+\ell_{3}+e_{3} \\
\ell_{2}+e_{2} & \sim 2+\ell_{3}+e_{3} .
\end{aligned}
$$

\footnotetext{
${ }^{5}$ This "switching behavior" was exploited in ref. [54] to generate an anarchical neutrino spectrum and large mixing angles.

${ }^{6}$ As a bonus, the $A_{11}^{e}$ term, responsible for generating an electron EDM, receives additional suppression.
} 
where the last two relations ensure the correct $e-\tau$ and $\mu-\tau$ mass ratios. A possible choice, satisfying also the unification conditions eq. (3.5), reads ${ }^{7}$

$$
\begin{aligned}
& q=(4,2,0), \quad u=(3,2,0), \quad e=(5,2,0), \\
& d=(5,0,0)+d_{3}, \quad \ell=(4,0,0)+d_{3}, \quad h_{d}^{\prime} \sim 5+d_{3},
\end{aligned}
$$

leading to Yukawas

$$
Y^{u} \sim\left(\begin{array}{ccc}
\epsilon^{7} & \epsilon^{6} & \epsilon^{0} \\
\epsilon^{5} & \epsilon^{4} & \epsilon^{2} \\
\epsilon^{3} & \epsilon^{2} & \epsilon^{0}
\end{array}\right), \quad Y^{d} \sim t_{\beta}\left(\begin{array}{ccc}
\epsilon^{\underline{\varepsilon}} & \epsilon^{7} & \epsilon^{7} \\
\epsilon^{8} & \epsilon^{5} & \epsilon^{5} \\
\epsilon^{8} & \epsilon^{3} & \epsilon^{3}
\end{array}\right), \quad Y^{e} \sim t_{\beta}\left(\begin{array}{ccc}
\epsilon^{8} & \epsilon^{\underline{\varepsilon}} & \epsilon^{7} \\
\epsilon^{8} & \epsilon^{5} & \epsilon^{3} \\
\epsilon^{8} & \epsilon^{5} & \epsilon^{3}
\end{array}\right) .
$$

The underlined exponents are the ones generated from the new contributions in the second term in eq. (4.9). One sees that only the down and the electron masses are affected by $h_{d}^{\prime}$. On the other hand, the A terms are given by

$$
A^{u} \sim Y^{u}, \quad A^{d} \sim t_{\beta}\left(\begin{array}{ccc}
\epsilon^{12} & \epsilon^{7} & \epsilon^{7} \\
\epsilon^{10} & \epsilon^{5} & \epsilon^{5} \\
\epsilon^{8} & \epsilon^{3} & \epsilon^{3}
\end{array}\right), \quad A^{e} \sim t_{\beta}\left(\begin{array}{ccc}
\epsilon^{12} & \epsilon^{9} & \epsilon^{7} \\
\epsilon^{8} & \epsilon^{5} & \epsilon^{3} \\
\epsilon^{8} & \epsilon^{5} & \epsilon^{3}
\end{array}\right) .
$$

The suppression in the 12 and 21 elements of $A^{e}$ is now sufficient for a slepton mass around $200 \mathrm{GeV}$. Notice that the FN models, even with multiple U(1)'s, have no analogue of this mechanism.

Notice that in order to forbid R-parity violating operators we need to impose R-parity as symmetry of the effective action. Once this is done, there are usually still dangerous dimension five operators. In our case, if the triplet Higgs fields are localized on the UV brane along with the doublets, these operators are naturally generated there, and we find

$$
\frac{1}{\Lambda_{\mathrm{UV}}} \epsilon^{q_{i}+q_{j}+q_{l}+l_{m}} Q_{i} Q_{j} Q_{k} L_{m} \quad, \quad \frac{1}{\Lambda_{\mathrm{UV}}} \epsilon^{u_{i}+u_{j}+d_{k}+e_{m}} U_{i} U_{j} D_{k} E_{m} .
$$

Due to the localization of the first two generations on the IR brane, we get an additional suppression, as for the UV localized Yukawas of the first two generations, which is enough in order to bring these operators into their experimental bounds [51].

\section{Conclusions}

Supersymmetric models with WFR reproduce the success of the FN models for fermion masses with mixings, alleviating at the same time their FCNC problems. Whereas from a $4 \mathrm{~d}$ perspective, the improvement in the quark sector is phenomenologically quite successful, in the leptonic sector there are still problems with $\mu \rightarrow e \gamma$. We however showed that in an extra dimensional realization similar to RS but with an IR brane of mass scale of the order of $M_{\mathrm{GUT}}$, with the first two generations composite (IR localized) and the third one

\footnotetext{
${ }^{7}$ Notice that this choice is $\mathrm{SU}(5)$-symmetric for the second and third generation, whereas there is a slight deviation from SU(5) symmetry for the first family. This could be realized, for example, by SU(5) breaking localized near the IR brane.
} 
elementary (UV localized), the problem can be elegantly solved by generating the electron mass by the strong coupling in the CFT sector. Indeed, the A-term for the electron is strongly suppressed since the supersymmetry breaking spurion field is elementary and the corresponding terms in the action (as well as the other soft breaking terms) are localized on the UV brane. More generally, the analogy between FN and WFR is precise in the warped 5d realization when all Yukawa couplings are elementary (UV localized), whereas strong coupling contributions (IR CFT contributions) add new structure compared to the FN setup. As a side comment, we notice that similarly, we can generate a $\mu$-term on the IR boundary with large suppression factor if $h_{u}^{\prime}, h_{d}^{\prime} \gg 0$ in the sense discussed in section 4 . This is of course useful only if for some reason such a term is absent on the UV brane.

We showed that whereas the FN gauge $\mathrm{U}(1)$ case is constrained by the various gauge anomaly cancelation conditions, in the WFR case most of these conditions re-emerged in section 3 as conditions for gauge coupling unification. More precisely, the same conditions for the corresponding parameters as the mixed anomaly conditions $A_{3} \sim \mathrm{U}(1)_{X} \mathrm{SU}(3)^{2}$, $A_{2} \sim \mathrm{U}(1)_{X} \mathrm{SU}(2)^{2}, A_{1} \sim \mathrm{U}(1)_{X} \mathrm{U}(1)_{Y}^{2}$ for the $\mathrm{U}(1)$ charges appear in the threshold corrections to the gauge couplings (3.1), (3.4). They are then constrained by the unification of the SM gauge couplings precisely in the same way as the U(1) charges by the universal GreenSchwarz anomaly cancelation conditions in the FN case. The mixed anomaly $\mathrm{U}(1)_{X}^{2} \mathrm{U}(1)_{Y}$ does not emerge in the WFR setup however and it is therefore still true that in the WFR case the "charges" $q_{i} \leftrightarrow c_{i}$ are less constrained than $\mathrm{U}(1)_{X}$ charges in the FN setup.

One should also mention that in the FN case $\mathrm{U}(1)_{X}$ can be broken to discrete symmetries $Z_{N}$ which can have nice features like suppressing proton decay at acceptable levels. There does not seem to be analog of this phenomenon in the WFR case.

On the side of the phenomenological predictions of the WFR scheme and the possibility of its experimental verification, one sees that FCNC effect are much more strongly suppressed than in the FN models. Thus, contrary to the predictions of the FN models, one does not expect the FCNC effects to be close to the present bounds (perhaps with exception of the muon decay). However, there is an interesting correlation between the supersymmetric models for flavour and the pattern of superpartner masses. The WFR scheme predicts all superpartner masses, except the stop masses, in terms of the gluino mass. In particular, also slepton masses are predicted in terms of the gluino mass.

Finally, it would be interesting to investigate the issue of flavor violation in F-theory models, where similarly there is an analog of the WFR of generating Yukawa hierarchies [55-59] and a different, gauged FN setup generating them [60-62].

\section{Acknowledgments}

We thank Tony Gherghetta and Claudio Scrucca for stimulating and helpful discussions. The work presented was supported in part by the European ERC Advanced Grant 226371 MassTeV, by the CNRS PICS no. 3059 and 4172, by the grants ANR-05-BLAN-0079-02, the PITN contract PITN-GA-2009-237920, the IFCPAR CEFIPRA programme 4104-2 and by the MNiSZW grant N N202 103838 (2010-2012). SP thanks the Institute for Advanced Studies at TUM, Munich, for its support and hospitality. 
Open Access. This article is distributed under the terms of the Creative Commons Attribution Noncommercial License which permits any noncommercial use, distribution, and reproduction in any medium, provided the original author(s) and source are credited.

\section{References}

[1] C.D. Froggatt and H.B. Nielsen, Hierarchy of Quark Masses, Cabibbo Angles and CP-violation, Nucl. Phys. B 147 (1979) 277 [SPIRES].

[2] Y. Nir and N. Seiberg, Should squarks be degenerate?, Phys. Lett. B 309 (1993) 337 [hep-ph/9304307] [SPIRES].

[3] M. Leurer, Y. Nir and N. Seiberg, Mass matrix models: The Sequel, Nucl. Phys. B 420 (1994) 468 [hep-ph/9310320] [SPIRES].

[4] L.E. Ibáñez and G.G. Ross, Fermion masses and mixing angles from gauge symmetries, Phys. Lett. B 332 (1994) 100 [hep-ph/9403338] [SPIRES].

[5] P. Binetruy and P. Ramond, Yukawa textures and anomalies, Phys. Lett. B 350 (1995) 49 [hep-ph/9412385] [SPIRES].

[6] P. Binetruy, S. Lavignac and P. Ramond, Yukawa textures with an anomalous horizontal Abelian symmetry, Nucl. Phys. B 477 (1996) 353 [hep-ph/9601243] [SPIRES].

[7] N. Irges, S. Lavignac and P. Ramond, Predictions from an anomalous U(1) model of Yukawa hierarchies, Phys. Rev. D 58 (1998) 035003 [hep-ph/9802334] [SPIRES].

[8] E. Dudas, S. Pokorski and C.A. Savoy, Yukawa matrices from a spontaneously broken Abelian symmetry, Phys. Lett. B 356 (1995) 45 [hep-ph/9504292] [SPIRES].

[9] Y. Nir, Gauge unification, Yukawa hierarchy and the mu problem, Phys. Lett. B 354 (1995) 107 [hep-ph/9504312] [SPIRES].

[10] E. Dudas, S. Pokorski and C.A. Savoy, Soft scalar masses in supergravity with horizontal $\mathrm{U}(1)_{X}$ gauge symmetry, Phys. Lett. B 369 (1996) 255 [hep-ph/9509410] [SPIRES].

[11] E. Dudas, C. Grojean, S. Pokorski and C.A. Savoy, Abelian flavour symmetries in supersymmetric models, Nucl. Phys. B 481 (1996) 85 [hep-ph/9606383] [SPIRES].

[12] P.H. Chankowski, K. Kowalska, S. Lavignac and S. Pokorski, Update on fermion mass models with an anomalous horizontal U(1) symmetry, Phys. Rev. D 71 (2005) 055004 [hep-ph/0501071] [SPIRES].

[13] W. Altmannshofer, A.J. Buras, S. Gori, P. Paradisi and D.M. Straub, Anatomy and Phenomenology of FCNC and CPV Effects in SUSY Theories, Nucl. Phys. B 830 (2010) 17 [arXiv:0909.1333] [SPIRES].

[14] R. Barbieri, G.R. Dvali and L.J. Hall, Predictions From A U(2) Flavour Symmetry In Supersymmetric Theories, Phys. Lett. B 377 (1996) 76 [hep-ph/9512388] [SPIRES].

[15] R. Barbieri, L.J. Hall and A. Romanino, Consequences of a U(2) flavour symmetry, Phys. Lett. B 401 (1997) 47 [hep-ph/9702315] [SPIRES].

[16] S.F. King and G.G. Ross, Fermion masses and mixing angles from SU(3) family symmetry, Phys. Lett. B 520 (2001) 243 [hep-ph/0108112] [SPIRES].

[17] S.F. King and G.G. Ross, Fermion masses and mixing angles from SU(3) family symmetry and unification, Phys. Lett. B 574 (2003) 239 [hep-ph/0307190] [SPIRES]. 
[18] Y. Kawamura and T. Kobayashi, Soft Scalar Masses in String Models with Anomalous U(1) symmetry, Phys. Lett. B 375 (1996) 141 [Erratum ibid. B 388 (1996) 867] [hep-ph/9601365] [SPIRES].

[19] Z. Lalak, S. Pokorski and G.G. Ross, Beyond MFV in family symmetry theories of fermion masses, JHEP 08 (2010) 129 [arXiv: 1006.2375] [SPIRES].

[20] A.E. Nelson and M.J. Strassler, Suppressing flavor anarchy, JHEP 09 (2000) 030 [hep-ph/0006251] [SPIRES].

[21] T. Kobayashi and H. Terao, Sfermion masses in Nelson-Strassler type of models: SUSY standard models coupled with SCFTs, Phys. Rev. D 64 (2001) 075003 [hep-ph/0103028] [SPIRES].

[22] A.E. Nelson and M.J. Strassler, Exact results for supersymmetric renormalization and the supersymmetric flavor problem, JHEP 07 (2002) 021 [hep-ph/0104051] [SPIRES].

[23] K.-w. Choi, D.Y. Kim, I.-W. Kim and T. Kobayashi, SUSY flavor problem and warped geometry, hep-ph/0301131 [SPIRES].

[24] K.-w. Choi, D.Y. Kim, I.-W. Kim and T. Kobayashi, Supersymmetry breaking in warped geometry, Eur. Phys. J. C 35 (2004) 267 [hep-ph/0305024] [SPIRES].

[25] Y. Nomura, M. Papucci and D. Stolarski, Flavorful Supersymmetry, Phys. Rev. D 77 (2008) 075006 [arXiv:0712 .2074] [SPIRES].

[26] Y. Nomura, M. Papucci and D. Stolarski, Flavorful Supersymmetry from Higher Dimensions, JHEP 07 (2008) 055 [arXiv: 0802 .2582] [SPIRES].

[27] S.J. Huber and Q. Shafi, Fermion Masses, Mixings and Proton Decay in a Randall-Sundrum Model, Phys. Lett. B 498 (2001) 256 [hep-ph/0010195] [SPIRES].

[28] S.J. Huber, Flavor violation and warped geometry, Nucl. Phys. B 666 (2003) 269 [hep-ph/0303183] [SPIRES].

[29] K. Agashe, G. Perez and A. Soni, B-factory signals for a warped extra dimension, Phys. Rev. Lett. 93 (2004) 201804 [hep-ph/0406101] [SPIRES].

[30] K. Agashe, G. Perez and A. Soni, Flavor structure of warped extra dimension models, Phys. Rev. D 71 (2005) 016002 [hep-ph/0408134] [SPIRES].

[31] G. Cacciapaglia et al., A GIM Mechanism from Extra Dimensions, JHEP 04 (2008) 006 [arXiv:0709.1714] [SPIRES].

[32] C. Csáki, C. Delaunay, C. Grojean and Y. Grossman, A Model of Lepton Masses from a Warped Extra Dimension, JHEP 10 (2008) 055 [arXiv: 0806. 0356] [SPIRES].

[33] C. Csáki, A. Falkowski and A. Weiler, A Simple Flavor Protection for RS, Phys. Rev. D 80 (2009) 016001 [arXiv: 0806.3757] [SPIRES].

[34] S. Casagrande, F. Goertz, U. Haisch, M. Neubert and T. Pfoh, Flavor Physics in the Randall-Sundrum Model: I. Theoretical Setup and Electroweak Precision Tests, JHEP 10 (2008) 094 [arXiv: 0807.4937 ] [SPIRES].

[35] F. Gabbiani, E. Gabrielli, A. Masiero and L. Silvestrini, A complete analysis of FCNC and $C P$ constraints in general SUSY extensions of the standard model,

Nucl. Phys. B 477 (1996) 321 [hep-ph/9604387] [SPIRES]. 
[36] D. Choudhury, F. Eberlein, A. Konig, J. Louis and S. Pokorski, Constraints on nonuniversal soft terms from flavor changing neutral currents, Phys. Lett. B 342 (1995) 180 [hep-ph/9408275] [SPIRES].

[37] G. D'Ambrosio, G.F. Giudice, G. Isidori and A. Strumia, Minimal flavour violation: An effective field theory approach, Nucl. Phys. B 645 (2002) 155 [hep-ph/0207036] [SPIRES].

[38] S. Davidson, G. Isidori and S. Uhlig, Solving the flavour problem with hierarchical fermion wave functions, Phys. Lett. B 663 (2008) 73 [arXiv:0711.3376] [SPIRES].

[39] G. Isidori, Y. Nir and G. Perez, Flavor Physics Constraints for Physics Beyond the Standard Model, arXiv: 1002.0900 [SPIRES].

[40] Particle Data Group collaboration, C. Amsler et al., Review of particle physics, Phys. Lett. B 667 (2008) 1 [SPIRES].

[41] H. Georgi, A.E. Nelson and A. Manohar, On The Proposition That All Fermions Are Created Equal, Phys. Lett. B 126 (1983) 169 [SPIRES].

[42] M.A. Shifman and A.I. Vainshtein, On holomorphic dependence and infrared effects in supersymmetric gauge theories, Nucl. Phys. B 359 (1991) 571 [SPIRES].

[43] V. Kaplunovsky and J. Louis, Field dependent gauge couplings in locally supersymmetric effective quantum field theories, Nucl. Phys. B 422 (1994) 57 [hep-th/9402005] [SPIRES].

[44] D. Poland and D. Simmons-Duffin, Superconformal Flavor Simplified, JHEP 05 (2010) 079 [arXiv:0910.4585] [SPIRES].

[45] A. Pomarol, Grand Unified Theories without the Desert, Phys. Rev. Lett. 85 (2000) 4004 [hep-ph/0005293] [SPIRES].

[46] W.D. Goldberger, Y. Nomura and D. Tucker-Smith, Warped supersymmetric grand unification, Phys. Rev. D 67 (2003) 075021 [hep-ph/0209158] [SPIRES].

[47] K. Agashe, R. Contino and R. Sundrum, Top compositeness and precision unification, Phys. Rev. Lett. 95 (2005) 171804 [hep-ph/0502222] [SPIRES].

[48] Y. Nomura, D. Poland and B. Tweedie, Holographic Grand Unification, JHEP 12 (2006) 002 [hep-ph/0605014] [SPIRES].

[49] L. Randall and R. Sundrum, A large mass hierarchy from a small extra dimension, Phys. Rev. Lett. 83 (1999) 3370 [hep-ph/9905221] [SPIRES].

[50] T. Gherghetta and A. Pomarol, Bulk fields and supersymmetry in a slice of AdS, Nucl. Phys. B 586 (2000) 141 [hep-ph/0003129] [SPIRES].

[51] R. Barbier et al., R-parity violating supersymmetry, Phys. Rept. 420 (2005) 1 [hep-ph/0406039] [SPIRES].

[52] M. Gabella, T. Gherghetta and J. Giedt, A gravity dual and LHC study of single-sector supersymmetry breaking, Phys. Rev. D 76 (2007) 055001 [arXiv:0704.3571] [SPIRES].

[53] O. Aharony, L. Berdichevsky, M. Berkooz, Y. Hochberg and D. Robles-Llana, Inverted Sparticle Hierarchies from Natural Particle Hierarchies, Phys. Rev. D 81 (2010) 085006 [arXiv: 1001.0637] [SPIRES].

[54] K. Agashe, T. Okui and R. Sundrum, A Common Origin for Neutrino Anarchy and Charged Hierarchies, Phys. Rev. Lett. 102 (2009) 101801 [arXiv:0810.1277] [SPIRES]. 
[55] C. Beasley, J.J. Heckman and C. Vafa, GUTs and Exceptional Branes in F-theory - II: Experimental Predictions, JHEP 01 (2009) 059 [arXiv:0806.0102] [SPIRES].

[56] A. Font and L.E. Ibáñez, Yukawa Structure from U(1) Fluxes in F-theory Grand Unification, JHEP 02 (2009) 016 [arXiv:0811.2157] [SPIRES]

[57] J.J. Heckman and C. Vafa, Flavor Hierarchy From F-theory, Nucl. Phys. B 837 (2010) 137 [arXiv:0811.2417] [SPIRES].

[58] S. Cecotti, M.C.N. Cheng, J.J. Heckman and C. Vafa, Yukawa Couplings in F-theory and Non-Commutative Geometry, arXiv:0910.0477 [SPIRES].

[59] J.P. Conlon and E. Palti, Aspects of Flavour and Supersymmetry in F-theory GUTs, JHEP 01 (2010) 029 [arXiv:0910.2413] [SPIRES].

[60] E. Dudas and E. Palti, Froggatt-Nielsen models from E8 in F-theory GUTs, JHEP 01 (2010) 127 [arXiv:0912.0853] [SPIRES].

[61] S.F. King, G.K. Leontaris and G.G. Ross, Family symmetries in F-theory GUTs, Nucl. Phys. B 838 (2010) 119 [arXiv: 1005.1025] [SPIRES].

[62] E. Dudas and E. Palti, On hypercharge flux and exotics in F-theory GUTs, JHEP 09 (2010) 013 [arXiv:1007.1297] [SPIRES]. 